\title{
Open-field behavior in mice: Analysis of maternal effects by means of ovarian transplantation'
}

J. C. DEFRIES, E. A. THOMAS, J. P. HEGMANN AND MORTOH W. WEIR

UNIVERSITY OF ILLINOIS

Ovaries from donors of each of two inbred strains (BALB/ cJ and $\mathrm{C} 57 \mathrm{BL} / 6 \mathrm{~J})$ were transplanted into $\mathrm{F}_{1}$ hybrid recipients. By appropriate matings it was thus possible to obtain inbred offspring carried by either hybrid or inbred mothers. Differences in the matemal environment were found to account for a relatively small proportion of the total variance in open-field behavior, but accounted for a relatively large proportion of the variance in body weight.

Broadhurst (1961) has discussed the general problem of the analysis of maternal effects on offspring behavior and has assessed the importance of the prenatal and postnatal maternal environment on openfield behavior in rats by means of reciprocal crossing and cross-fostering, respectively. In general, little evidence for the presence of directional maternal effects was found.

One difficulty in assessing maternal effects by means of the reciprocal crossing of two inbred strains is that all resulting progeny are hybrid. These offspring may be less susceptible than are inbreds to environmental sources of variation (cf., Falconer, 1960). The alternative techniques of ovarian and ova transplantation have the advantage of avoiding this and other difficulties associated with the use of reciprocal crossing as a technique for the assessment of maternal effects. Ovaries from inbred donors may be transplanted into hybrid recipients, and by appropriate matings, it is possible to obtain inbred offspring of the same strains which have been carried by females of different genotypes (inbred vs. hybrid) and inbred offspring of different strains which have been carried by females of the same genotype (hybrid). With transplantation of fertilized ova across and within inbred strains, all possible combinations of maternal and offspring genotypes may be obtained. However, this procedure is technically difficult and does not allow for recovery from surgical intervention prior to pregnancy.

The primary objective of the present study was to assess the role of the maternal environment on the open-field behavior of inbred offspring, utilizing the technique of ovarian transplantation.

\section{Method}

Ovaries from 24 donors of each of two inbred strains of mice (BALB/cJ and C57BL/6J) were transplanted bilaterally into $F_{1}$ hybrid females. The surgical procedure outlined by Palm (1961) for orthotopic grafts employing surgical removal of host ovaries was followed.

The mating system was such that inbred progeny were carried by either hybrid or inbred mothers. Ten days after surgery, hybrid females carrying transplanted ovaries from BALB donors were mated to inbred $B A L B$ males and hybrid females bearing C57BL ovaries were mated to inbred C57BL males. Offspring from these matings are designated $B / H$ and $\mathrm{C} / \mathrm{H}$, respectively (see Table 1 ). Within-strain matings of inbred males to unoperated inbred females produced control offspring. The resulting inbred BALB offspring carried by inbred BALB mothers and inbred C57BL offspring carried by C57BL mothers are designated $\mathrm{B} / \mathrm{B}$ and $\mathrm{C} / \mathrm{C}$, respectively.

At $40 \pm 5$ days of age, all offspring from first litters were tested in a brightly lighted open fleld for $3 \mathrm{~min}$ on each of two successive days. Activity and defecation data were obtained and two-day totals were used as each S's score. The apparatus and testing proceaure have been described elsewhere (DeFries et al, 1966). Body weight data in grams were also obtained.

The offspring and maternal strain combinations that result from the system of mating employed are presented in Table 1. Due to histocompatibility differences between these two strains of mice, tissue grafts across inbred strains are not successful. As a consequence, two of the six combinations shown in Table 1 are not possible with ovarian transplantation. Thus, since the main effect due to strain of mother is not orthogonal with that due to strain of offspring in this experiment, analysis of variance was deemed inappropriate, and $t$ tests of statistical significance were used to test for the presence of maternal effects by means of the following comparisons: $B / B$ vs $B / H$, and $\mathrm{C} / \mathrm{C}$ vs $\mathrm{C} / \mathrm{H}$.

\section{Resulis and Discussion}

From the 48 matings of hybrid recipient females,

Table 1. Combinations of Offspring and Matemal Strains Using Ovarian Transplantation*

\begin{tabular}{lccc} 
Strain of Offspring & BALB/CJ & $\begin{array}{c}\text { Strain of Mother } \\
\text { C57BL/6J }\end{array}$ & Hybrid \\
\hline BALB/CJ & $\mathrm{B} / \mathrm{B}$ & -- & $\mathrm{B} / \mathrm{H}$ \\
$\mathrm{C} 57 \mathrm{BL} / 6 \mathrm{~J}$ & -- & $\mathrm{C} / \mathrm{C}$ & $\mathrm{C} / \mathrm{H}$ \\
\hline
\end{tabular}

* The first letter symbolizes strain of offspring and the second letter symbolizes strain of mother, i.e., $B / H$ refers to $B A L B / c J$ offspring carried by $F_{1}$ hybrid mothers, etc. 
Table 2. Mean Open-field Activity and Defecation Scores and Body Weight of Inbred Progeny Carried by Inbred and Hybrid Mothers ${ }^{a}$

\begin{tabular}{lccccc} 
& \multicolumn{3}{c}{ Combinations of Offspring and Maternal Strains } \\
& $\mathrm{B} / \mathrm{B}$ & $\mathrm{B} / \mathrm{H}$ & $\mathrm{C} / \mathrm{C}$ & $\mathrm{C} / \mathrm{H}$ Variance \\
\hline Activity & 4.08 & 4.30 & 15.86 & 16.02 & 6.41 \\
Defecation & 2.71 & 2.95 & 1.17 & 1.09 & 0.30 \\
Body Weight & 16.22 & 18.24 & 16.56 & 18.93 & 2.53 \\
N & 72 & 79 & 71 & 39 & \\
\hline
\end{tabular}

a Unweighted means of average scores of male and female offspring. Symbols for combinations of offspring and maternal strains are explained in Table 1. Mean activity scores were obtained from transformed data. where each subject's score is the square root of the total activity over the two-day test. Mean defecation scores were obtained from transformed data, where $x=1$ total boluses + $1 / 2)^{1 / 2}$. Body weight data were adjusted for differences in litter size within each subclass.

$b$ Within sub-class variance, pooled across sex of offspring, strain of mother and strain of offspring.

32 first litters were obtained. However, since reconstitution of remnants of the host ovary may occur (cf., Palm, 1961), second and third litters were also obtained where possible in order to maximize the probability that offspring of hybrid mothers did not result from the fertilization of ova resulting from residual host germinal tissue. Inbred BALB offspring are albino, whereas inbred C57BL offspring are black; thus, the presence of offspring with inappropriate coat colors in the first or any subsequent litter resulted in the exclusion of the data from that female's first litter. Only three first litters were discarded on the basis of this criterion. Thus, it appears that the technical proficiency was such that the risk of production of host ova was low in this experiment.

The resulting mean transformed open-field activity and defecation scores and body weight data from the 29 litters produced by hybrid mothers and 32 litters from inbred mothers are summarized in Table 2. Weight data were adjusted for litter size, due to a significant correlation between body weight and number of offspring per litter $(r=-.18, p<.01$, df= 253).

Comparisons of data from $B / B$ vs $B / H$ and $C / C$ vs $\mathrm{C} / \mathrm{H} \mathrm{Ss}$, pooled across sex of offspring, were utilized to test for the presence of maternal effects. With regard to open-field activity, no evidence for the presence of a maternal effect is indicated ( $t<1$ for both comparisons). With regard to defecation, $\mathrm{C} / \mathrm{C}$ vs $\mathrm{C} / \mathrm{H}$ was also nonsignificant $(t<1)$; however, $B / B$ vs $B / H$ was significant $(t=2.69, d f=149, p<.01)$.
As expected, a large and highly significant maternal effect on body weight was found $(B / B$ vs $B / H, t=7.82$, $\mathrm{df}=149, \mathrm{p}<.001 ; \mathrm{C} / \mathrm{C}$ vs $\mathrm{C} / \mathrm{H}, \mathrm{t}=7.46, \mathrm{df}=108, \mathrm{p}<$ $.001)$. Thus, evidence for a general maternal effect on the two behavioral measures was found only in the case of defecation in BALB offspring. This effect is small in comparison with that on body weight, a characteristic previously known to be influenced by the maternal environment (Falconer, 1965). Since no cross-fostering was employed in the present experiment, it should be noted that maternal effects could arise during either prenatal or postnatal development.

The effect due to strain of offspring (B vs C, pooled across strain of mother and sex of offspring) was large and highly significant for both open-field activity and defecation $(t=35.79$ and 23.87 , respectively). With regard to body weight, this effect was smaller, but significant $(t=2.37, d f=253, p<.025)$. The effect due to sex of offspring was significant with regard to both open-field activity and body weight $(t=2.10, p<.05 ; t=12.33, p<.001$, respectively), and approached significance for defecation $(t=1.91$, df $=253, \mathrm{p}<.10$ ).

The results of this experiment indicate that the effects of the prenatal and postnatal maternal environment account for a relatively small proportion of the total variance in open-field activity and defecation, especially when compared to that due to strain of offspring. These results, in agreement with those of Broadhurst (1961), also suggest that it may not be necessary to control for the effects of the prenatal and postnatal maternal environment in studies regarding the inheritance of open-field behavior.

\section{References}

Broadhurst, P. L. Analysis of maternal effects in the inheritance of behaviour. Anim. Behav., 1961, 9, 129-141.

DeFries, J. C., Hegmann, J. P., \& Weir, M. W. Open-field behavior in mice: Evidence for a major gene effect mediated by the visual system. Science, 1966, 154, 1577-1579.

Falconer. D. S. Introduction to quantitative genetics. New York: The Ronald Press, 1960, 270-272.

Falconer, D. S. Maternal effects and selection response. In Genetics today, Vol. 3, Proc. XI Int. Cong. Genetics, The Hague 1963. Oxford: Pergammon Press, 1965, 763-774.

Palm, J. Transplantation of ovarian tissue. In R. E. Billingham \& W. K. Silvers (Eds.), Transplantation of tissues and cells. Philadelphia: The Wistar Institute Press, 1961. Pp. 49-56.

Note

1. Supported in part by NIH grant GM-12486. 